\title{
Does Every Quasar Harbor A Blazar?
}

\author{
Feng Ma and Beverley J. Wills \\ McDonald Observatory, University of Texas, Austin, TX 78712; feng@astro.as.utexas.edu, \\ bev@astro.as.utexas.edu
}

Received __; accepted _ 


\begin{abstract}
Assuming there is a blazar type continuum in every radio-loud quasar, we find that the free-free heating due to the beamed infrared continuum can greatly enhance collisionally excited lines, and thus explain the stronger CIV $\lambda 1549$ line emission observed in radio loud quasars. We further predict that the CIV line should show variability not associated with observed continuum or Ly $\alpha$ variability.
\end{abstract}

Subject headings: galaxies: nuclei-quasars: emission lines-quasars: general 


\section{Introduction}

The dichotomy between radio-loud and radio-quiet QSOs remains one of the greatest puzzles in studies of AGN. While their optical-UV spectra appear similar, quasars (we use the term "quasar" for radio loud QSO) have CIV $\lambda 1549$ line emission $~ 50 \%$ stronger compared with radio-quiet QSOs (Francis, Hooper, \& Impey 1993). Marziani et al. (1996) find that the luminosities of CIV and $\mathrm{H} \beta$ are strongly correlated in radio-quiet QSOs, but only weakly correlated in quasars. Some observed line profile differences could also be the result of an additional beamed optical-UV synchrotron continuum in quasars, but not in radio-quiet QSOs (Wills et al. 1993; Francis et al. 1993).

Unified schemes for quasars hold that there is a beamed synchrotron continuum in every quasar (e.g., Urry \& Padovani 1995). If the beam is toward the observer, the quasar is seen as a blazar. Blazars are known to be highly variable on time scales from days to years. For example, BL Lacertae brightened by 3 magnitudes in $\mathrm{R}$ band during the outburst in the summer of 1997 (Bloom et al. 1997). The outburst lasted for a few months, and during the outburst it shows intraday and even intrahour variation of $\sim 1$ magnitude.

Bregman et al. (1986) found that the Ly $\alpha$ line flux varied in proportion to the continuum flux density in the blazar 3C446. Stephens \& Miller (1984) found that the CIII] $\lambda 1909$ line flux varied with the continuum in 3C446 and suggested that the beamed

continuum might affect the emission line spectra. Koratkar et al. (1998) found no variation in the flux of Lya emission line in HST spectra of the blazar 3C 279 while the continuum varied by a factor of 50. Cohen et al. (1987) found possible Mg II line variability in the blazar AO $0235+164$. Corbett et al. (1996) found the $\mathrm{H} \alpha$ line in BL Lac to be variable, but not as a result of the beamed continuum. The emission line variability in blazars is not easy to measure because of the large contribution from the variable continuum. Most previous studies of AGN spectral variability have focused on objects known to have 
large continuum variability and concentrated on the emission line variations following the observed continuum variations (e.g., Maoz et al. 1994).

In this Letter we investigate the interactions between the beamed synchrotron radiation and the BELR. Although the beam may illuminate only a small portion of the Broad Emission Line Region (BELR), it can provide a large flux of infrared photons that heat the gas by the free-free mechanism (Ferland et al. 1992), greatly enhancing the CIV $\lambda 1549$ line emission. This enhanced emission can significantly contribute to the line flux from the entire BELR, explaining the stronger line emission seen in quasars. The CIV line emission is thus expected to vary in response to the rapid and large amplitude variations of the blazar continuum, although we may not see the continuum variation if it is beamed away from us.

\section{Additional Beamed Continua in Quasars}

Theoretically, a simple blazar synchrotron continuum is beamed with a half opening

angle $\theta=\sin ^{-1}(1 / \gamma)$ for a Lorentz factor $\gamma$. The fraction of the sky area illuminated by the beam is $1 / 2 \gamma^{2}$, or $\sim 2 \%$ for $\gamma=5$, and $\sim 5 \%$ for $\gamma=3$. The real beaming angle could be larger (Lind \& Blandford 1985), and can be estimated from the observed fraction of blazars among quasars as a whole (e.g., Urry \& Padovani 1995).

If the beamed synchrotron continuum originates closer to the central black hole than the BELR ( $\sim 0.1$ pc in luminous quasars, see e.g., Netzer 1990) then it may affect the emission line spectrum. In NGC 1275 the VLBI jet probably originates within 0.1 pc at $43 \mathrm{GHz}$ (Krichbaum et al. 1992). The higher frequency synchrotron radiation of interest $\left(\sim 10^{12} \mathrm{~Hz}\right)$ is likely to originate closer to the black hole. Some theoretical models predict infrared synchrotron emission originating $\sim 0.1 \mathrm{pc}$ from the black hole and that lower frequency emission originates farther away from the center (see e.g., Marscher 1996). 
The beamed synchrotron continuum provides a high flux of IR photons because of its steep spectrum, which extends to mm wavelengths. In contrast, the normal QSO continuum provides only weak IR flux, with much of the observed IR emission almost certainly arising from dust beyond the BELR (Barvainis, Antonucci, \& Coleman 1992). Moreover, if the observed IR continuum in radio-quiet QSOs were within BELR distances it would significantly overpredict the observed CIV strength.

\section{Models}

Ferland et al. (1992) showed that a high flux of IR photons incident on BELR clouds could enhance the flux of the CIV and OVI emission lines by free-free heating of the BELR. As discussed above, a strong IR central continuum is unlikely in radio-quiet QSOs. However, for BELR gas exposed exposed to the beamed synchrotron radiation, free-free heating is the most important heating process.

The heating rate is proportional to (e.g., Netzer 1990)

$$
F_{\nu} e^{-\tau_{\nu}(f f)} T_{e}^{-1 / 2} N_{e} N_{i} \nu^{-3}\left(1-e^{-h \nu / k T_{e}}\right) .
$$

Collisional excitation results in an important emission line cooling process in optically thick BELR gas, and the rate is proportional to

$$
N_{e} \Omega_{i j} e^{-E_{i j} / k T_{e}} T_{e}^{-1 / 2},
$$

where $\Omega_{i j}$ is the collision strength. For CIV $\lambda 1549, E_{i j} \sim 8 \mathrm{eV}$, and the cooling rate increases with temperature. At $\sim 16 \mathrm{eV}$, this line intensity stops increasing with $T_{e}$ because the collision cross sections become smaller for higher velocity electrons. Roughly speaking, the IR heating leads to a higher electron temperature, which results in stronger CIV $\lambda 1549$.

We use a fixed $L_{\mathrm{agn}}=10^{46} \mathrm{erg} \mathrm{s}^{-1}$ for an isotropic AGN continuum and use the spectral energy distribution (SED) of Mathews \& Ferland (1987) except that the submillimeter 
break is at $10 \mu \mathrm{m}$. The beamed continuum is conservatively assumed to have $f_{\nu} \propto \nu^{-1}$ from $10^{-3.5}$ to $10^{-2}$ Ryd, and $f_{\nu} \propto \nu^{-2}$ from $10^{-2}$ to $10^{6}$ Ryd. At lower energies, $f_{\nu} \propto \nu^{2.5}$. We assume that the beamed continuum has constant intensity within the small opening angle $2 \theta$, and use $L_{\text {beam }}$ to represent the apparent luminosity as seen by an observer within the beam. Adding $L_{\text {beam }}$ essentially does not change the ionization parameter because of the steepness of the spectrum.

Solar abundances are assumed for the BELR gas. The calculations are done using CLOUDY (Ferland 1996). We first try a simple BELR model "A" resembling a model given by Ferland \& Persson (1989). They use a fixed hydrogen density $n_{H}=10^{9.5} \mathrm{~cm}^{-3}$, and a fixed ionization parameter $U=10^{-0.5}$. Because we are using $L_{\text {agn }}=10^{46} \mathrm{erg} \mathrm{s}^{-1}$ for the input continuum, $U=10^{-0.5}$ constrains the cloud distance to $r=10^{17.67} \mathrm{~cm}$. The column density for the cloud is set to be $10^{25.5} \mathrm{~cm}^{-2}$.

In the more realistic model "B" we follow the model of Goad, O'Brien, \& Gondhalekar (1993), in which discrete clouds are distributed from $10^{17} \mathrm{~cm}$ to $10^{19} \mathrm{~cm}$ from the continuum source. The density starts from $10^{13} \mathrm{~cm}^{-3}$, and has a power law distribution $n \propto r^{-2}$. The column density is set to be $N(r) \propto r^{-4 / 3}$, starting from $10^{23} \mathrm{~cm}^{-2}$ at the inner radius. The differential covering factor varies as $\propto r^{-1 / 6}$, to fulfill the mass conservation of the clouds. The calculated emission line luminosities are integrated from inner to outer radius.

\section{Results}

In Tables 1 and 2 we list the calculated emission line luminosities for models $\mathrm{A}$ and $\mathrm{B}$. For the $\mathrm{H} \beta$ line we give the logarithm of luminosity in units of $\mathrm{erg} \mathrm{s}^{-1}$, and the other line luminosities are given as the ratio to $\mathrm{H} \beta$.

Column 2 is the result for $L_{\text {agn }}=10^{46} \mathrm{erg} \mathrm{s}^{-1}$ with no $L_{\text {beam }}$; column 3 is for $L_{\text {agn }}=10^{46}$ 
$\mathrm{erg} \mathrm{s}^{-1}$ mixed with $L_{\text {beam }}=10^{46} \mathrm{erg} \mathrm{s}^{-1}$, and $L_{\text {beam }}$ affects only $2 \%$ of the sky area. A small covering factor $(\sim 0.1)$ for the clouds is assumed everywhere in the sky, including in the beam. Columns 4 and 5 correspond to $L_{\text {beam }}=10^{46.5}$ and $10^{47} \mathrm{erg} \mathrm{s}^{-1}$. The observed line ratios in column 6 are taken from Netzer (1990).

From Tables 1 and 2 it can be seen that among strong lines, the CIV $\lambda 1549$ is enhanced by $\sim 50 \%$ when $L_{\text {beam }}=10^{47} \mathrm{erg} \mathrm{s}^{-1}$, while hydrogen lines show little change. The CIII $\lambda 977$ is also strongly dependent on the IR heating. These two lines actually set up an upper limit of $L_{\text {beam }} \sim 10^{47} \mathrm{erg} \mathrm{s}^{-1}$, otherwise they would be too strong. However, we note that CIII $\lambda 977$ is difficult to observe because of intervening absorption, especially at higher redshifts. Model A predicts a slight enhancement of OVI $\lambda 1035$ and NV $\lambda 1240$. We do not consider their enhancement in model $\mathrm{B}$, because the strengths of these two lines are not well predicted.

In Fig. 1 we plot for model $\mathrm{A}$ the abundance ratio $\mathrm{C}^{3+} / \mathrm{C}$, and the electron temperature versus the depth into the cloud. As expected, the $\mathrm{C}^{3+}$ density is not strongly affected by the beamed continuum, and CIV $\lambda 1549$ is an effective coolant.

In Fig. 2, we give cloud surface temperature and various line luminosities versus radius in model B. The free-free heating is more important at small radii, since it is proportional to the photon flux as shown in eq. (1), while photoelectric heating by ionizing photons is determined by the hydrogen number density and the recombination coefficient and does not depend explicitly on photon flux (Ferland et al. 1992).

Some line profiles with and without a beamed continuum are compared in Fig. 3. We show the profiles here primarily for the purpose of showing the enhancement of integrated emission lines flux due to the beamed continuum, though affecting only a small portion of the BELR clouds. The BELR cloud distribution and velocity field are probably anisotropic with velocities having a strong disk component in addition to a random component (e.g., 
Wills \& Browne 1986). For simplicity, here we consider only spherically distributed clouds with a Keplerian velocity field, and the velocity direction is assumed to be random. The maximum cloud velocity is $10^{4} \mathrm{~km} \mathrm{~s}^{-1}$ at $r=10^{17} \mathrm{~cm}$. The flat tops of the profiles come from the lowest gas velocity, $10^{3} \mathrm{~km} \mathrm{~s}^{-1}$ at $r=10^{19} \mathrm{~cm}$.

\section{Discussion}

The IR-optical continuum of the synchrotron radiation is steep, hence the fraction of hydrogen ionizing photons it can offer is small compared with the normal AGN continuum. In addition, the beam illuminates only a small fraction of the BELR clouds, while the emission lines we see are integrated over all the clouds. Consequently, the effect on the $\mathrm{H} \alpha$ and Ly $\alpha$ lines is negligible. For collisionally excited lines such as CIV $\lambda 1549$, the line emissivity can be enhanced by over two orders of magnitude in the beam, and the line intensity integrated over the whole BELR consequently increases by a factor of $~ 50 \%$.

If there is a beamed blazar type continuum in every radio loud quasar, we should be able to see CIV line variability not associated with observed continuum variability or Ly $\alpha$ variability. This phenomenon could well have escaped detection in previous observations because high redshift quasars have not been extensively monitored. Also, this phenomenon may be seen only when the blazar continuum is in the outburst stage. We are planning observations to search for CIV $\lambda 1549$ line variability among $z \sim 2.5$ quasars, which are thought to be less variable compared with low luminosity AGNs. If the CIV line variability at the level $\sim 50 \%$ is observed, while the lines for comparison (Ly $\alpha, \mathrm{CIII}] \lambda 1909$ or $\mathrm{MgII}$ $\lambda 2798$ ) do not change significantly, it will be strong support for our models and for quasar unified schemes. In addition, the disk-wind model (e.g., Emmering, Blandford, \& Shlosman 1992) for the BELR will be ruled out, since it does not include any gas in the polar regions. 
We also expect quasars to have statistically stronger CIV $\lambda 1549$. The strength of CIV $\lambda 1549$ depends strongly on the blazar continuum, and should not be correlated well with $\mathrm{H} \beta$ line strength as shown by Marziani et al. (1996). The intensity variation may be seen in the line core or line wings, and thus offer information about the gas kinematics in the polar regions, e.g., whether the gas clouds have lower velocity or have large radial velocities driven by the jet.

In reality, the line emission depends on the details of BELR geometry. If the line is strongly beamed backward (e.g., Ferland et al. 1992), we may not be able to see the enhanced CIV emission when the angle between the line of sight and the beam is large. However, the excited lines are at least more isotropic than the beamed continuum, and we do expect to see the line variability without seeing the beam.

If the phenomenon suggested by us is not detected, this will still give strong constraints on models. Three possibilities are left. First, the IR synchrotron radiation originates outside the BELR. Second, the observed blazar continuum variability may be caused by a change of the jet direction rather than a change in strength of the beam (e.g., Abraham \& Carrara 1998). Third, there is no broad line emitting gas in the polar regions. The last possibility would favor the disk-wind model and may rule out the bloated star model (e.g., Alexander \& Netzer 1994).

Finally we note that if the BELR is made of small clouds confined by a hot medium (Krolik, McKee, \& Tarter 1981), then the jet might destroy the clouds. However, if the BELR is made of bloated stars, the jet may enhance the mass loss rate of the stars and increase the amount of broad line emitting gas in the beam.

We thank Greg Shields for helpful discussions, and Gary Ferland for advice and making his code CLOUDY available. We also thank the referee, Ski Antonucci, for valuable 
comments. This work is supported by NASA LTSA grant NAG5-3431. 


\section{REFERENCES}

Abraham, Z., \& Carrara, E. A. 1998, ApJ, 496, 172

Alexander, T., \& Netzer, H. 1994, MNRAS, 270, 781

Barvainis, R., Antonucci, R., \& Coleman, P. 1992, ApJ, 399, L19

Bloom, S. D. et al. 1997, ApJ, 490, L145

Bregman, J. N., Glassgold, A. E., Huggins, P. J., \& Kinney, A. L. 1986, ApJ, 301, 698

Corbett, E. A. et al. 1996, MNRAS, 281, 737

Cohen, R. D., Smith, H. E., Junkkarinen, V. T., \& Burbidge, E. M. 1987, ApJ, 318, 577

Emmering, R. T., Blandford, R. D., \& Shlosman, I. 1992, ApJ, 385, 460

Ferland, G. J., \& Persson, S. E. 1989, ApJ, 347, 656

Ferland, G. J., Peterson, B. M., Horne, K., Welsh, W. F., \& Nahar, S. 1992, ApJ, 387, 95

Ferland, G. J. 1996, Hazy, a Brief Introduction to Cloudy, University of Kentucky Department of Physics and Astronomy Internal Report

Francis, P. J., Hooper, E. J., \& Impey, C. D. 1993, AJ, 106, 2

Goad, M. R., O’Brien, P. T., \& Gondhalekar, P. M. 1993, MNRAS, 263, 149

Koratkar, A., Pian, E., Urry, C. M., \& Pesce, J. 1998, ApJ, 492, 17

Krichbaum, T. P. et al. 1992, A\&A, 260, 33

Krolik, J. H., McKee, C. F., \& Tarter, C. B. 1981, ApJ, 249, 422

Lind, K. R., \& Blandford, R. D. 1985, ApJ, 295, 358 
Maoz, D., Smith, P., Jannuzi, B., Kaspi, S., \& Netzer, H. 1994, ApJ, 421, 34

Marscher, A. P. 1996, in ASP. Conf. Proc. 100, Energy Transport in Radio Galaxies and Quasars, e.d. Hardee, P. E., Bridle, A. H., \& Zensus, J. A. (San Francisco: ASP), 45

Marziani, P., Sulentic, J. W., Dultzin-Hacyan, D., Calvani, M., \& Moles, M. 1996, ApJS, 104,37

Mathews, W. G., \& Ferland, G. J. 1987, ApJ, 323, 456

Netzer, H. 1990, in Active Galactic Nuclei, ed. R. D. Blandford, H. Netzer, \& L. Woltjer (Berlin:Springer), 57

Stephens, S. A., \& Miller, J. S., 1984, BAAS, 16, 1007

Urry, C. M., \& Padovani, P. 1995, PASP, 107, 803

Wills, B. J., \& Browne, I. W. A., 1986, ApJ, 302, 56

Wills, B. J., Brotherton, M. S., Fang, D., Steidel, C. C., \& Sargent, W. W. 1993, ApJ, 415, 563 


\section{Figure Captions}

Fig. 1 Temperature and $\mathrm{C}^{3+} / \mathrm{C}$ profiles inside the cloud in model $\mathrm{A}$. The solid lines correspond to the case with $L_{\mathrm{agn}}=10^{46} \mathrm{erg} \mathrm{s}^{-1}$, and the dashed lines correspond to the case with $L_{\text {agn }}=10^{46} \mathrm{erg} \mathrm{s}^{-1}$ mixed with $L_{\text {beam }}=10^{47} \mathrm{erg} \mathrm{s}^{-1}$.

Fig. 2 Surface temperature of clouds and emission line luminosity versus radius in model B. The solid lines correspond to the case with $L_{\mathrm{agn}}=10^{46} \mathrm{erg} \mathrm{s}^{-1}$, and the dashed lines correspond to the case with $L_{\mathrm{agn}}=10^{46} \mathrm{erg} \mathrm{s}^{-1}$ mixed with $L_{\text {beam }}=10^{46.0}, 10^{46.5}, 10^{47.0} \mathrm{erg} \mathrm{s}^{-1}$ respectively. $L_{\text {beam }}$ and $L_{\text {agn }}$ both illuminate $4 \pi$ steradian.

Fig. 3 Emission line profiles in model B. The solid lines correspond to the case with $L_{\mathrm{agn}}=10^{46} \mathrm{erg} \mathrm{s}^{-1}$, and the dashed line corresponds to the case with $L_{\mathrm{agn}}=10^{46} \mathrm{erg}$ $\mathrm{s}^{-1}$ mixed with $L_{\text {beam }}=10^{47} \mathrm{erg} \mathrm{s}^{-1}$. $L_{\text {beam }}$ illuminates only $2 \%$ of the clouds. The fluxes of different lines are arbitrary. 
Table 1. Model A

\begin{tabular}{|c|c|c|c|c|c|}
\hline \multirow[b]{2}{*}{ Line } & \multicolumn{4}{|c|}{$L_{\text {beam }}$} & \multirow[b]{2}{*}{ Obs. } \\
\hline & 0 & $10^{46}$ & $10^{46.5}$ & $10^{47}$ & \\
\hline Ly $\alpha 1216$ & 25.3 & 25.3 & 25.3 & 25.5 & $8-15$ \\
\hline CIV $\lambda 1549$ & 11.4 & 11.9 & 13.1 & 16.0 & $5-8$ \\
\hline CIII] $\lambda 1909$ & 6.0 & 6.1 & 6.5 & 7.5 & $2-4$ \\
\hline CIII $\lambda 977$ & 0.9 & 0.9 & 1.2 & 2.6 & $<1$ \\
\hline $\mathrm{H} \beta 4861$ & 43.59 & 43.60 & 43.61 & 43.61 & 1 \\
\hline $\mathrm{H} \alpha 6563$ & 2.3 & 2.3 & 2.3 & 2.3 & $4-6$ \\
\hline HeI $\lambda 5876$ & 0.2 & 0.2 & 0.2 & 0.2 & $0.1-0.2$ \\
\hline HeII $\lambda 1640$ & 1.1 & 1.1 & 1.1 & 1.1 & 0.6 \\
\hline HeII $\lambda 4686$ & 0.1 & 0.1 & 0.1 & 0.1 & 0.1 \\
\hline NIV] $\lambda 1486$ & 0.7 & 0.8 & 0.8 & 1.0 & 0.7 \\
\hline MgII $\lambda 2798$ & 1.2 & 1.2 & 1.3 & 1.3 & 3 \\
\hline SiIV,OIV] $\lambda 1400$ & 1.6 & 1.7 & 1.9 & 2.7 & 1.3 \\
\hline OIII] $\lambda 1665$ & 3.4 & 3.5 & 3.8 & 5.0 & 0.5 \\
\hline NIII] $\lambda 1750$ & 0.9 & 0.9 & 1.0 & 1.4 & 0.4 \\
\hline OVI $\lambda 1035$ & 3.0 & 3.2 & 3.5 & 4.2 & 3 \\
\hline NV $\lambda 1240$ & 1.0 & 1.1 & 1.1 & 1.3 & 3 \\
\hline
\end{tabular}


Table 2. Model B

\begin{tabular}{|c|c|c|c|c|c|}
\hline \multirow[b]{2}{*}{ Line } & \multicolumn{4}{|c|}{$L_{\text {beam }}$} & \multirow[b]{2}{*}{ Obs. } \\
\hline & 0 & $10^{46}$ & $10^{46.5}$ & $10^{47}$ & \\
\hline Ly $\alpha 1216$ & 37.4 & 37.4 & 37.4 & 37.6 & $8-15$ \\
\hline CIV $\lambda 1549$ & 5.9 & 6.4 & 7.4 & 10.1 & $5-8$ \\
\hline CIII] $\lambda 1909$ & 2.4 & 2.4 & 2.4 & 2.5 & $2-4$ \\
\hline CIII $\lambda 977$ & 1.1 & 1.4 & 2.2 & 5.1 & $<1$ \\
\hline $\mathrm{H} \beta 4861$ & 43.99 & 44.00 & 44.00 & 44.01 & 1 \\
\hline $\mathrm{H} \alpha 6563$ & 4.7 & 4.7 & 4.7 & 4.6 & $4-6$ \\
\hline HeI $\lambda 5876$ & 0.7 & 0.7 & 0.7 & 0.7 & $0.1-0.2$ \\
\hline HeII $\lambda 1640$ & 2.4 & 2.4 & 2.4 & 2.4 & 0.6 \\
\hline HeII $\lambda 4686$ & 0.3 & 0.3 & 0.3 & 0.3 & 0.1 \\
\hline NIV] $\lambda 1486$ & 0.1 & 0.1 & 0.1 & 0.1 & 0.7 \\
\hline MgII $\lambda 2798$ & 9.7 & 9.8 & 9.9 & 10.2 & 3 \\
\hline SiIV,OIV] $\lambda 1400$ & 1.4 & 1.6 & 1.9 & 2.9 & 1.3 \\
\hline OIII] $\lambda 1665$ & 1.1 & 1.2 & 1.2 & 1.5 & 0.5 \\
\hline NIII] $\lambda 1750$ & 0.3 & 0.3 & 0.3 & 0.4 & 0.4 \\
\hline OVI $\lambda 1035$ & $10^{-3}$ & 0.002 & 0.003 & 0.01 & 3 \\
\hline NV $\lambda 1240$ & 0.02 & 0.03 & 0.05 & 0.1 & 3 \\
\hline
\end{tabular}




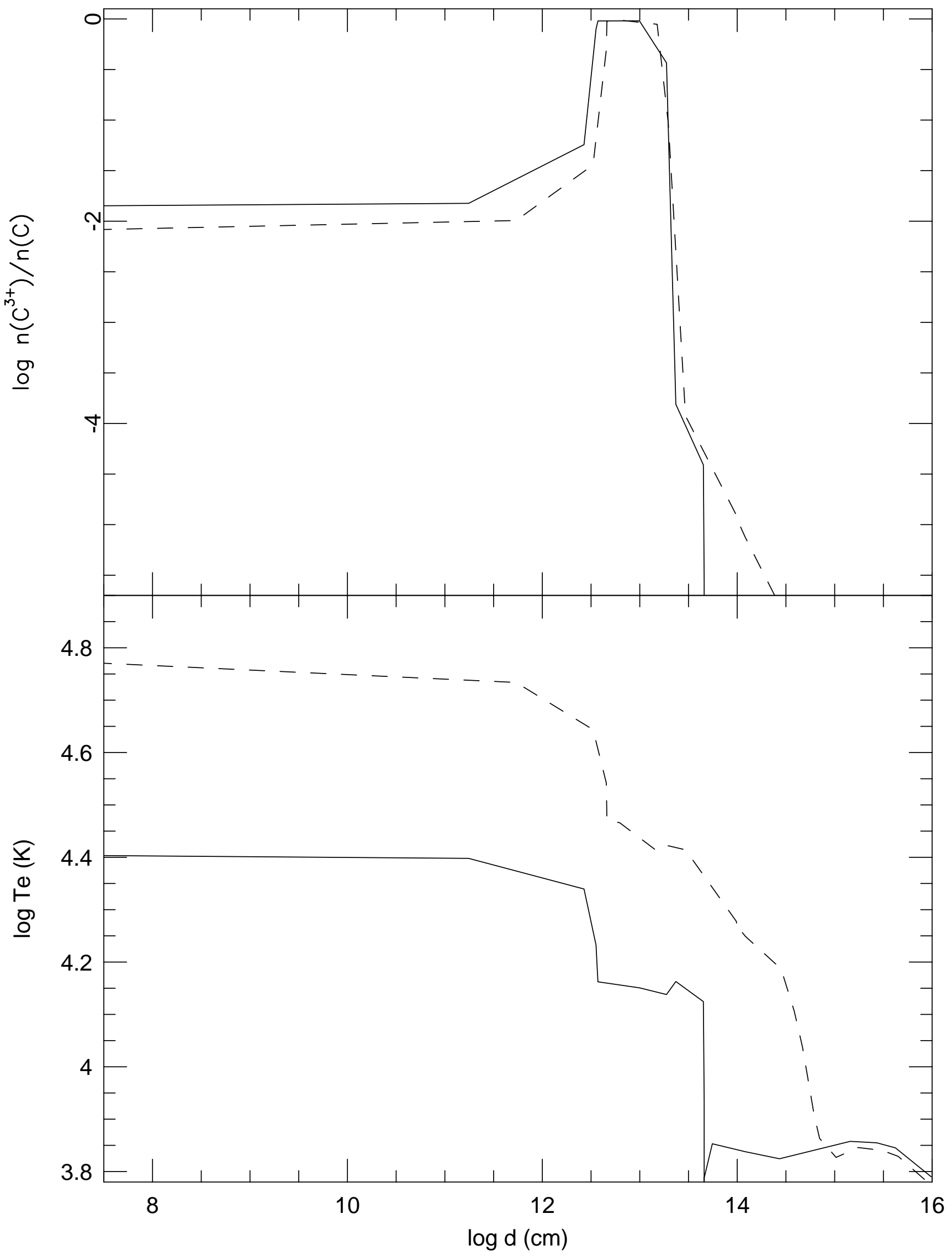



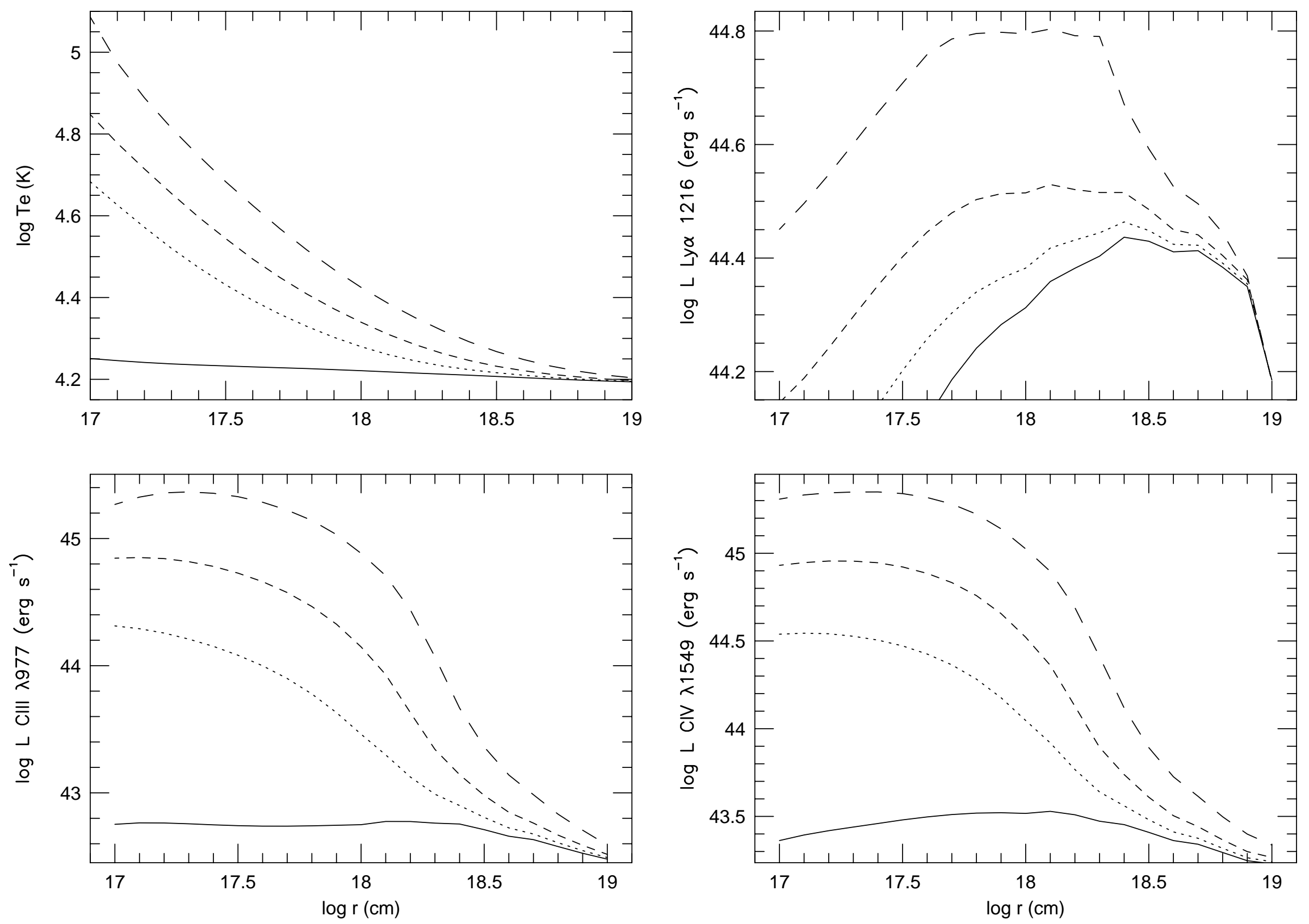

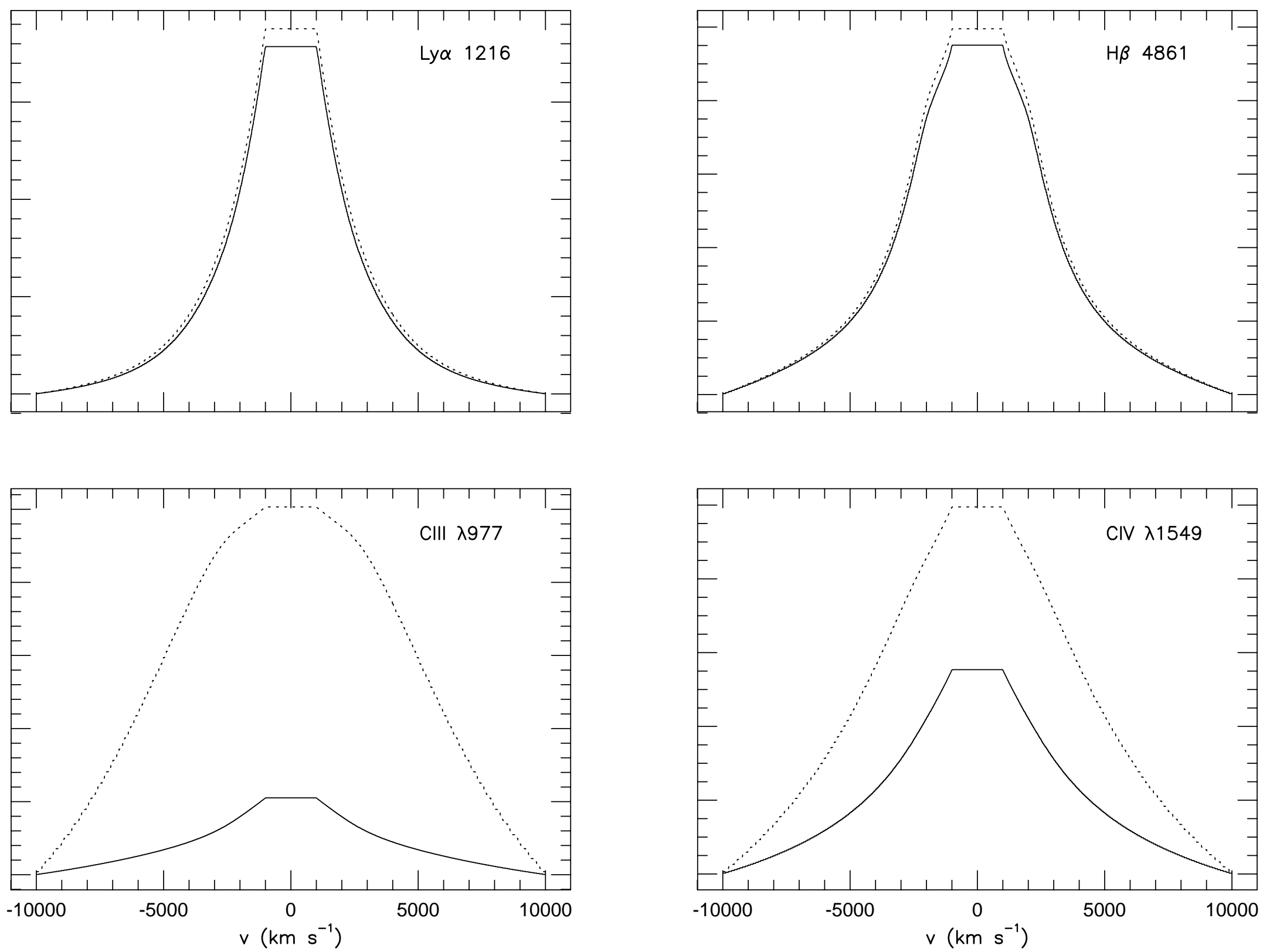